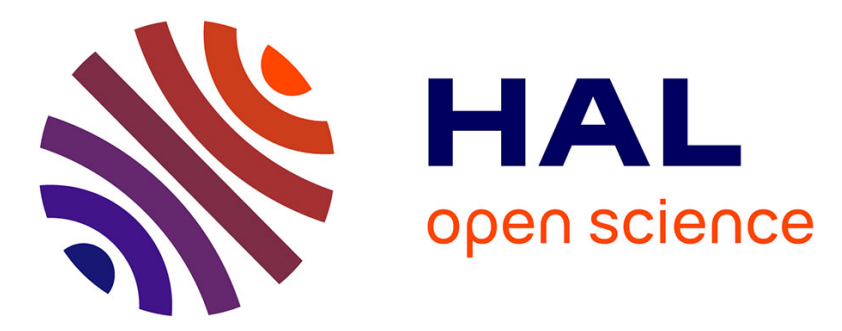

\title{
IMPORTANCE DE LA DURÉE DE TARISSEMENT DANS LE CYCLE DE PRODUCTION D'UNE VACHE LAITIÈRE
}

J.-J. Lauvergne, B. Vissac, J. Poly, J. Delage

\section{- To cite this version:}

J.-J. Lauvergne, B. Vissac, J. Poly, J. Delage. IMPORTANCE DE LA DURÉE DE TARISSEMENT DANS LE CYCLE DE PRODUCTION D'UNE VACHE LAITIÈRE. Le Lait, 1958, 38 (378), pp.481491. hal-00928224

\author{
HAL Id: hal-00928224 \\ https://hal.science/hal-00928224
}

Submitted on 1 Jan 1958

HAL is a multi-disciplinary open access archive for the deposit and dissemination of scientific research documents, whether they are published or not. The documents may come from teaching and research institutions in France or abroad, or from public or private research centers.
L'archive ouverte pluridisciplinaire HAL, est destinée au dépôt et à la diffusion de documents scientifiques de niveau recherche, publiés ou non, émanant des établissements d'enseignement et de recherche français ou étrangers, des laboratoires publics ou privés. 


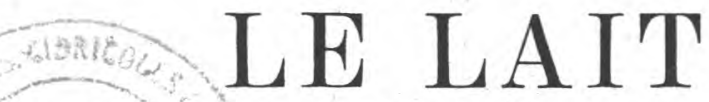

REVUE GENERALE DES QUESTIONS LAITIERES

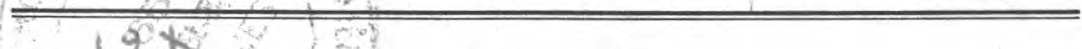

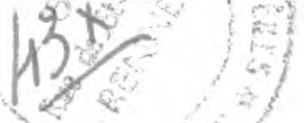

Mémoires originaux :

J.-J. LAUVERGNE, B. VISSAC, J. Poly, J. Delage. Importance de la durée de tarissement dans le cycle de production d'une vache laitière.

J.-M. DE MAN. - La teneur en gaz et son influence sur les propriétés du beurre. .

G. Curli et D. Coppini. Le brome dans le lait . . .

SOMMAIRE

REVUE :

G. Génin. - Le lait dans le monde. . . . . . .

\section{Bibliographie analytique :}

$1^{\circ}$ Les livres . . . . . . . 509

$2^{\circ}$ Journaux, Revues, Sociétés savantes . . . . . . 518

$3^{\circ}$ Brevets . . . . . . 566
Bulletin bibliographique :

$1{ }^{\circ}$ Journaux, Revues, Sociétés savantes . . . . . 571

$2^{\circ}$ Brevets . . . . . 577

Documents et informations :

Résultat de la lutte contre la tuberculose bovine en 1957.

Un peu de l'histoire du fromage de Brie . . . . . . 582

L'Institut "Zanelli " de Reggio Emilia et le nouveau Centre Expérimental " Pellegrino Spallanzani ». . . 589

Annuaire national du lait. 1958. 8e édition. . . . 591

Cours de Techniciens de l'Alimentation des Animaux domestiques . . . . 592

\section{MÉMOIRES ORIGINAUX (1)}

\section{IMPORTANCE DE LA DURÉE DE TARISSEMENT DANS}

\section{LE CYCLE DE PRODUCTION D'UNE VACHE LAITIËR}

par

J.-J. LAUVERGNE, B. VISSAC, J. POLY, J. DELAGE

Plan du mémoire:

1. Les motifs de cette étude.

2. Les travaux antérieurs.
3. Notre étude :
A. Matériel de travail.
B. Méthodes et résultats.

(1) Reproduction interdite sans indication de source. 


\section{I. - Les motifs de cette étude}

Pour eomparer à des fins de sélection les performances de production des vaches, enregistrées dans une population donnée, il faut connaître en détail les influences respectives des différents facteurs susceptibles de faire varier ces productions laitières, comme le mois de vêlage, l'intervalle entre le vêlage et la fécondation, le numéro d'ordre de la lactation, la durée de tarissement.

Ainsi, plusieurs études biométriques ont cherché à préciser quantitativement l'action de la période sèche sur la lactation suivante :

Du point de vue physiologique, on sait que la femelle a besoin d'une phase de repos pour lui permettre de reconstituer ses réserves et notamment ses réserves minérales, avant de commencer un nouveau cycle de production. L'importance de ce fait a été particulièrement bien soulignée par ForBes et coll.[2] à propos du métabolisme phosphocalcique de la vache.

De telles recherches peuvent permettre de préciser la durée optimum de tarissement, et, par suite, de renseigner l'éleveur sur les conditions dans lesquelles il doit exploiter son troupeau pour en obtenir une rentabilité maximum.

Nous exposerons successivement :

- Les principaux travaux antérieurs, qui nous permettront de connaître l'évolution des méthodes utilisées dans les recherches concernant l'influence de la durée de tarissement sur la production laitière suivante;

- Les résultats de notre étude et les renseignements pratiques que nous pouvons en tirer.

Nous conviendrons de représenter respectivement par :

- D. T., la durée de tarissement ;

- D. L., la durée de lactation;

- P. L., la production laitière.

\section{II. - Les travaux antérieurs}

Parmi les nombreux auteurs qui ont étudié les variations de la P. L. au cours d'une lactation en fonction de la D. T. précédente, il convient de signaler le travail de Lörtscher [6]. Ce chercheur fournit une expression mathématique de la courbe de régression de la P. L. (Y) sur la D. T. (X) :

$$
\mathrm{Y}=\mathrm{a}+\mathrm{b} \mathrm{X}+\mathrm{c} \mathrm{X}^{2}+\mathrm{d} \log \mathrm{X} \text { (Graphique 1). }
$$

Il semble, d'après lui, que chez les vaches frisonnes, les $\mathrm{D}$. T. inférieures à 40 jours soient suivies de lactations dont les P. L. sont inférieures d'environ $10 \%$ à la P. L. maximum ; au-delà de 80 jours, 


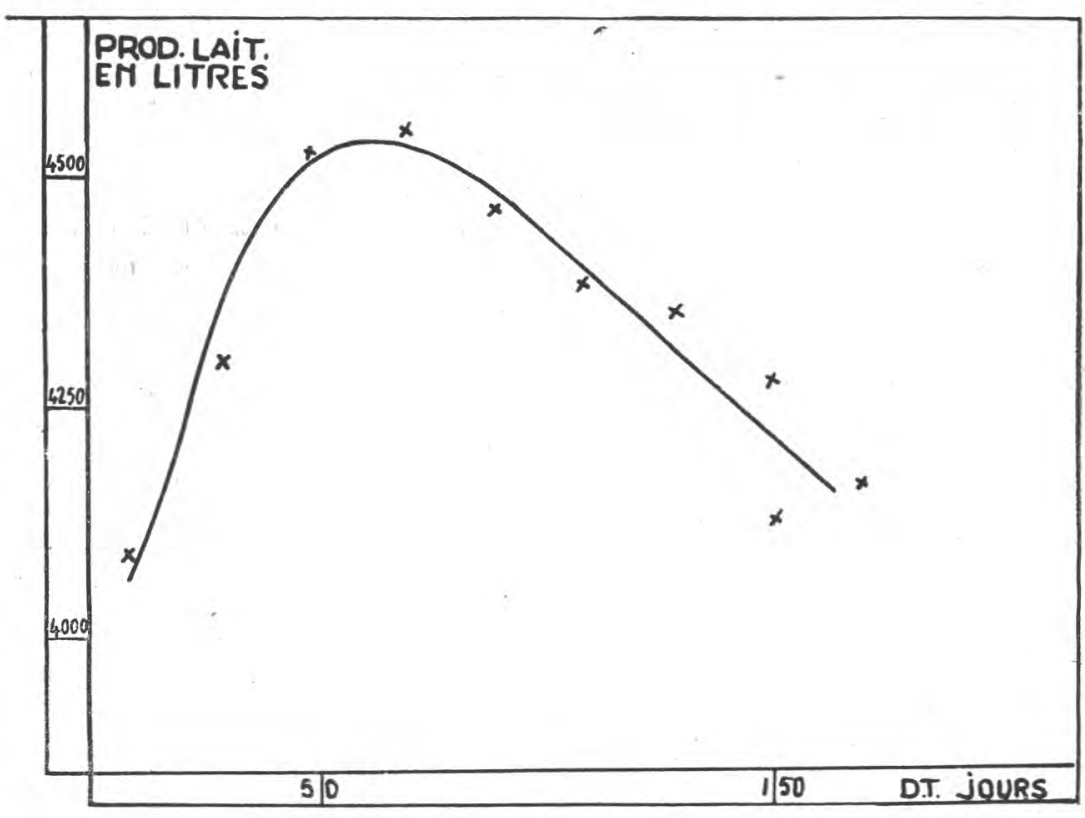

Fig. 1. - Courbe de Lörtscher

un accroissement de $\mathrm{D}$. T. s'accompagne d'une légère diminution de production attribuable surtout, selon lui, à des dépôts graisseux qui se forment au niveau de la mamelle lorsque la période sèche est trop prolongée.

De récents travaux de Swanson [10] confirment cette interprétation physiologique; ainsi, des génisses trop engraissées avant vêlage ont des productions moindres en première et deuxième lactations, par suite d'un développement insuffisant du tissu mammaire sécréteur, résultant d'une extension du tissu adipeux, alors qu'extérieurement leur pis apparaît bien, et même prématurément, formé.

SANDERs [9] ne travaille que sur des données de P. L. et D. T. corrigées statistiquement afin d'éliminer l'influence des principales causes de variation : mois de vêlage, intervalle vêlage-fécondation précédent et âge. Il est en effet logique de penser que ces facteurs peuvent intervenir pour modifier la relation étudiée. Dans cet esprit, et contrairement à la plupart des autres chercheurs, SANDERS tente d'apprécier l'influence, pour une vache déterminée, de la D. T. précédente sur la P. L. suivante, en exprimant les performances de production de la vache adulte en pourcentage de la valeur de P. L. correspondant à la D. T. la plus faible. Il néglige cependant dans son étude, de faire la part des facteurs individuels 
dans les variations des D. T., part qui est presque aussi importante que dans les variations de P. L., puisqu'elle se ohiffre entre 15 et $25 \%$ de la variance totale (Johansson et Hansson [4]).

SANDers trouve ainsi que la courbe de régression de la P. L. ( $Y$ en $\%$ ) sur la D. T. précédente ( $\mathrm{X}$ en jours) s'apparente à une exponentielle d'équation :

$$
\mathrm{Y}=129-33,9 \mathrm{e}^{-0,1625 \mathrm{x}}
$$

Il observe en fait que, lorsque la D. T. est inférieure à deux mois, la P. L. est sensiblement réduite; au delà de cette limite par contre, la P. L. s'accroît faiblement avec la D. T. (graphique 2). Tous ces résultats sont admis par A.-M. Leroy [5] qui signale une augmentation de production négligeable (de l'ordre de $4 \%$ ) à la suite de D. T. de deux cents jours, par rapport à des performances correspondant à des périodes sèches de deux mois.

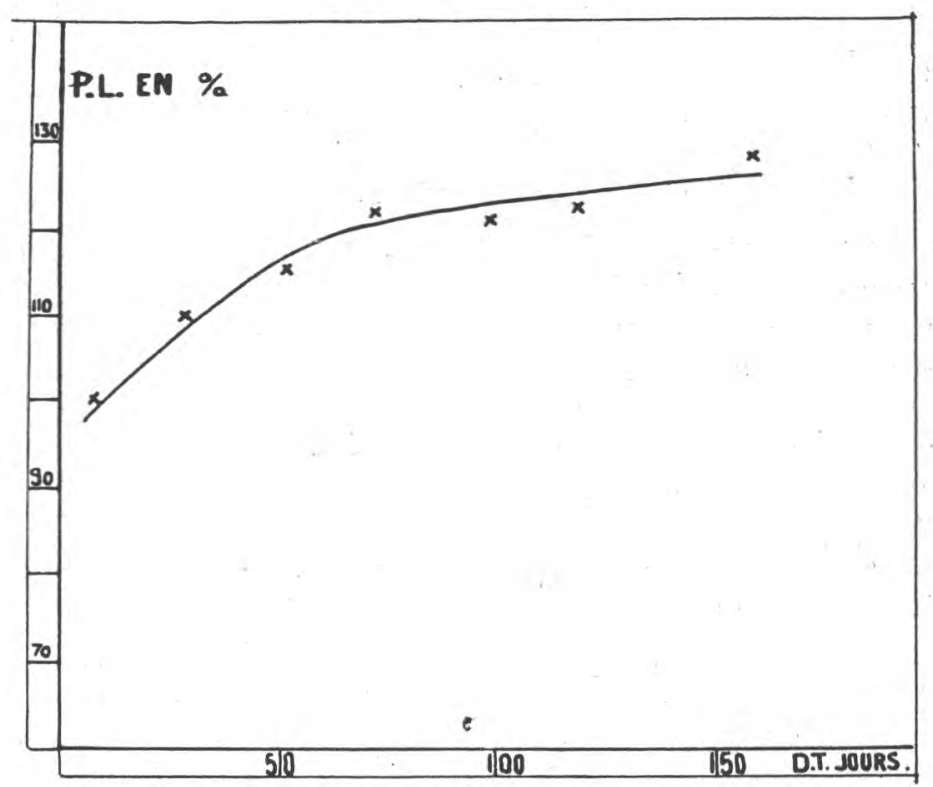

Fig. 2. - Courbe de Sanders.

Johansson et Hansson [4] utilisent dans leurs recherches la production de matière grasse, au cours d'une lactation de référence de trois cents jours, corrigée en fonction des facteurs de variation suivants : âge, mois de vêlage, intervalle de vêlage précédent et intervalle de vêlage correspondant. La D. T. est corrigée elle, en fonction de l'intervalle de vêlage dans laquelle elle est comprise. 
Cette façon d'opérer est critiquable à plusieuŕs égards ; en effet :

- Le fait de ne considérer que des lactations de référence implique au départ que la durée du cycle de production n'est pas modifiée par les variations de D. T. précédente. Nous verrons que cette supposition peut être infirmée;

- L'application d'un trop grand nombre de facteurs de correction risque de fausser la vraie relation entre les deux variables étudiées et d'ôter ainsi aux conclusions une partie de leur signification pratique.

L'idée originale de ces deux auteurs a été d'analyser l'action de la D. T. sur la production à l'échelon du troupeau, de la vache et au cours de ses différents cycles de production, et de montrer que ces influences s'exprimaient par des courbes de variation distinctes (graphique 3) présentant un maximum de production laitière pour des D. T. de cinquante à soixante jours.

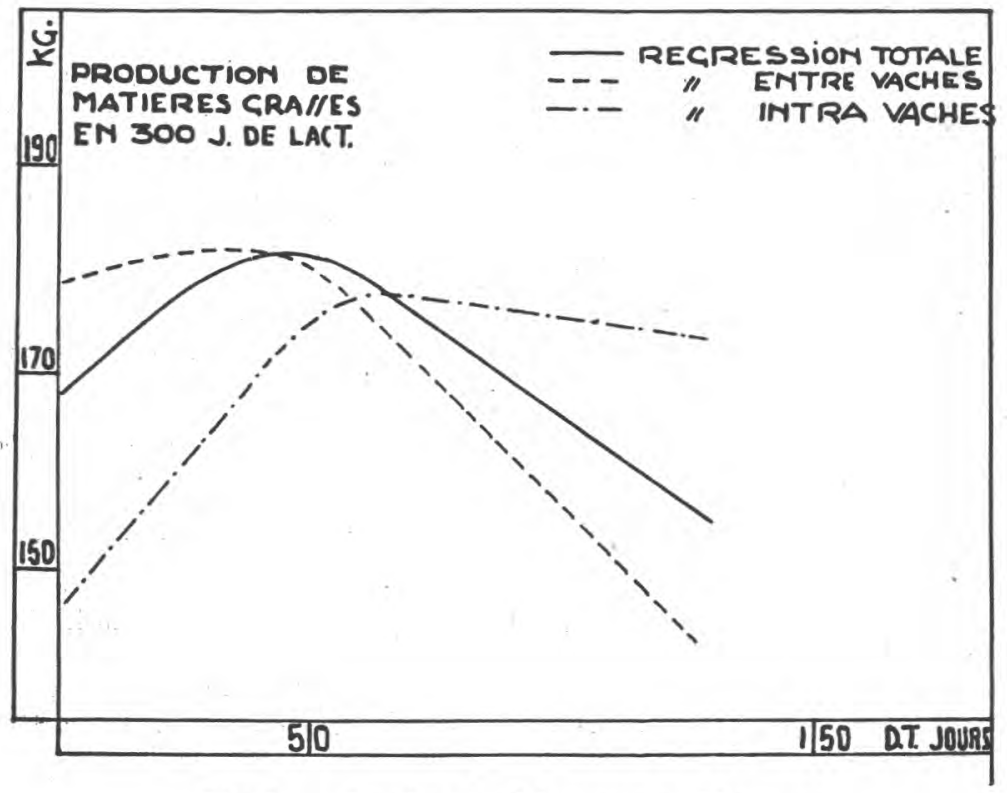

Fig. 3. - Courbes de Johansson et Hansson

Il est clair, d'après ce résultat, que la décroissance de P. L. observée au delà de cet optimum, par de nombreux auteurs, dont LöRTSCHER, est surtout due à des différences entre les aptitudes de chaque individu ; on sait que les vaches ayant présenté une courte durée de tarissement au cours du cycle de production précédent, le doivent à leur forte persistance, qui a toute chance de se mani- 
fester au cours du cycle de production suivant, par une plus longue durée de lactation.

\section{III. - Notre étude}

\section{A. Matériel de travail}

Cette étude se situe dans un eadre plus vaste de recherches, concernant l'influence des facteurs de milieu sur la P. L., effectuées depuis 1948 sur les données provenant du Syndicat de contrôle laitier de Seine-et-Marne. Elle a porté sur 1.960 lactations se rapportant à 835 vaches françaises frisonnes pie noires exploitées dans trente et un élevages de ce département. Nous avons retenu toutes les performances contrôlées de 1950 à 1954 . Notre échantillon de travail a été entièrement choisi au hasard ; nous l'avons analysé statistiquement en appliquant les règles de l'analyse de la covariance définies par SNEDECOR et Ostle.

\section{B. Méthodes et résultats}

Nous n'avons appliqué aucune correction à notre population de données, de taille relativement trop limitée pour nous permettre d'étudier systématiquement des facteurs de correction valables pour les principales sources de variation des deux critères considérés.

Nous pensons d'ailleurs que toute recherche sur cette question devrait être poursuivie sur des valeurs brutes de P. L. et de D. T., au cours des différentes saisons de vêlage, ou de différents cycles de production par exemple.

Pour aborder le problème, nous avons utilisé deux voies d'approche :

- analyse de covariance de la D. T. précédente avec la P. L. suivante ;

- détermination de la forme de la courbe de régression de la P. L. sur la D. T. précédente.

a) Analyse de covariance de la D. T. précédente avec la P. L. de la lactation suivante.

Nous nous sommes attachés à l'analyse chez les animaux adultes: troisième lactation et au delà (PoLY et coll. [7]) de la covariance des D. T. précédentes sur les P. L. suivantes (tableau I).

Les coefficients de corrélation obtenus ne sont pas significatifs, mais se classent respectivement dans le même ordre que ceux obtenus par JoHANsSON et HANsSON en ce qui concerne les liaisons totales, entre vaches, intra-vaches (Tableau I). Il est surtout 
TABLEAU I

\begin{tabular}{|c|c|c|c|c|c|}
\hline \multirow{3}{*}{$\begin{array}{l}\text { Sources } \\
\text { de } \\
\text { variation }\end{array}$} & \multirow{3}{*}{$\begin{array}{l}\text { Nombre } \\
\text { de degrés } \\
\text { de liberté }\end{array}$} & \multicolumn{3}{|c|}{ Cœfficients de eorrélation } & \multirow{2}{*}{ 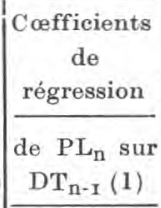 } \\
\hline & & entre $\mathrm{PL}_{\mathrm{n}}$ & et $\mathrm{DT}_{\mathrm{n}-\mathrm{I}}(1)$ & $\begin{array}{l}\text { entre } \mathrm{DL}_{\mathrm{n}} \\
\text { et } \mathrm{DT}_{\mathrm{n}-\mathrm{z}}(2)\end{array} \mid$ & \\
\hline & & $\begin{array}{l}\text { Nos } \\
\text { résultats }\end{array}$ & $\begin{array}{l}\text { Johansson } \\
\text { et Hansson }\end{array}$ & $\begin{array}{l}\text { Nos } \\
\text { résultats }\end{array}$ & $\begin{array}{l}\text { Nos } \\
\text { résultats }\end{array}$ \\
\hline Variation : & & & & & \\
\hline totale $\ldots \ldots \ldots$ & 446 & $-0,038$ & $-0,215$ & $-0,008$ & $-1,046$ \\
\hline entre vaches .... & 147 & $-0,152$ & $-0,401$ & $-0,111$ & $-4,050$ \\
\hline $\begin{array}{l}\text { entre troupeaux } \\
\text { entre vaches }\end{array}$ & 29 & $\div 0,019$ & $-0,616$ & $-0,089$ & $+0,818$ \\
\hline intra-troupeaux. & 119 & $-0,168$ & $-0,365$ & $-0,117$ & $-5,322$ \\
\hline résiduelle ...... & 298 & $+0,053$ & $+0,132$ & $-0,059$ & $+1,514$ \\
\hline
\end{tabular}

(1) $\mathrm{PL}^{\mathrm{t}}$ et $\mathrm{DT}^{\mathrm{t}}-/$ = production laitière de la lactation en cours et durée de tarissement précédente.

(2) $\mathrm{DL}^{t}$ et $\mathrm{DT}^{\mathrm{t}}$ - / = durée de la lactation en cours et durée de tarissement précédente.

intéressant de constater que les régressions linéaires de P. L. sur D. T. diffèrent suivant que l'on considère la relation globale $(b=-1,046)$, la liaison entre vaches $(b=-4,050)$ ou la liaison intra-vaches $(b=+1,514)$.

Dans le deuxième cas, il est logique de penser que les femelles à durée de tarissement longue ont des lactations courtes et moins persistantes, donc des productions laitières plus faibles.

Une liaison linéaire positive intra-vaches exprimerait, par contre, que pour une vache type de la population, un allongement de la période de repos mammaire entraîne en moyenne une augmentation de production dans la lactation suivante et inversement.

On peut se demander en particulier si la durée de tarissement ne modifie pas indirectement la production laitière en agissant sur les variations de durée de la lactation qu'elle précède. Nous avons, dans ce but, calculé les coefficients de corrélation entre deuxième $D$. T. et troisième $D$. L., troisième D. T. et quatrième D. L., quatrieme D. T. et cinquième D. L. qui sont respectivement de :

$$
-0,218-0,187-0,116
$$

les deux premiers sont significatifs, le troisième à la limite du seuil de signification de $5 \%$.

Nous avons cherché ensuite à préciser la nature de cette relation par une analyse de covariance, de données correspondant à 
des vaches adultes classées par étable, et par vache intra-étables (Tableau I).

Il semble donc, à la lumière de ces résultats, que la relation entre la D. T. et la D. L. suivante explique en grande partie la liaison entre vaches qui est apparue entre D. T. et P. L.

Toutefois, une analyse vraiment complète de cette question nécessite l'étude des courbes réelles de régression de la $P$. L sur la D. T. précédente.

De faibles coefficients de corrélation tels que ceux que nous avons trouvés peuvent être dûs, en effet, à un manque de linéarité des relations.

b) Courbes de régression des P. L. en cours sur les D. T. précédentes.

Nous avons tracé la courbe de régression des P. L. sur les D. T. précédentes pour l'ensemble de notre population de données en considérant séparément :

- les D. T. consécutives à la première lactation et les P. L. en deuxième lactation;

- les couples de données correspondant aux autres cycles de production.

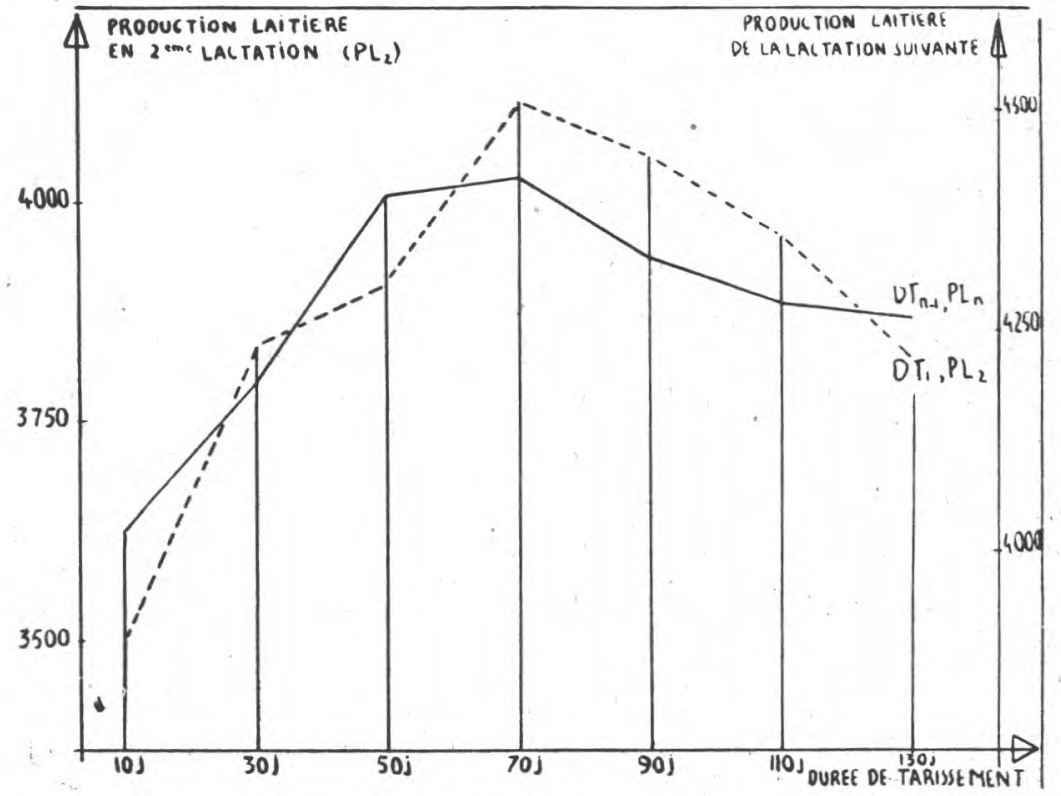

Fig. 4. - Influence de la durée de tarissement sur la production laitière de la lactation suivante 
Cette distinction nous permet de faire abstraction des principales variations dues à l'âge. Le graphique 4 indiquant la succession des valeurs moyennes de P. L., par classe de D. T., montre que la courbe de régression présente, dans les deux cas, un maximum pour des D. T. comprises entre soixante et quatre-vingts jours; on remarque également que les P. L. en deuxième lactation sont plus faibles lorsque les D. T. précédentes sont inférieures à quarante jours; cette limite est, par contre, ramenée à deux mois pour les autres lactations. La décroissance qui s'amorce au delà du maximum n'a pu, faute de données, être précisée pour les D. T. supérieures à cent cinquante jours.

Dans le but de donner la véritable expression de la relation intra-vaches entre la D. T. précédente et la P. L. suivante, nous avons enfin considéré un lot de 148 vaches dont nous connaissons les valeurs de ces deux séries de données pour les troisième, quatrième et cinquième lactations. Les variations de P. L. par classe de D. T. ont été calculées sur l'ensemble de la population et pour les moyennes individuelles des 148 vaches; on peut ainsi représenter graphiquement les courbes de régression générale et entre vaches (graphique 5). A partir de ces deux courbes, nous avons schématisé

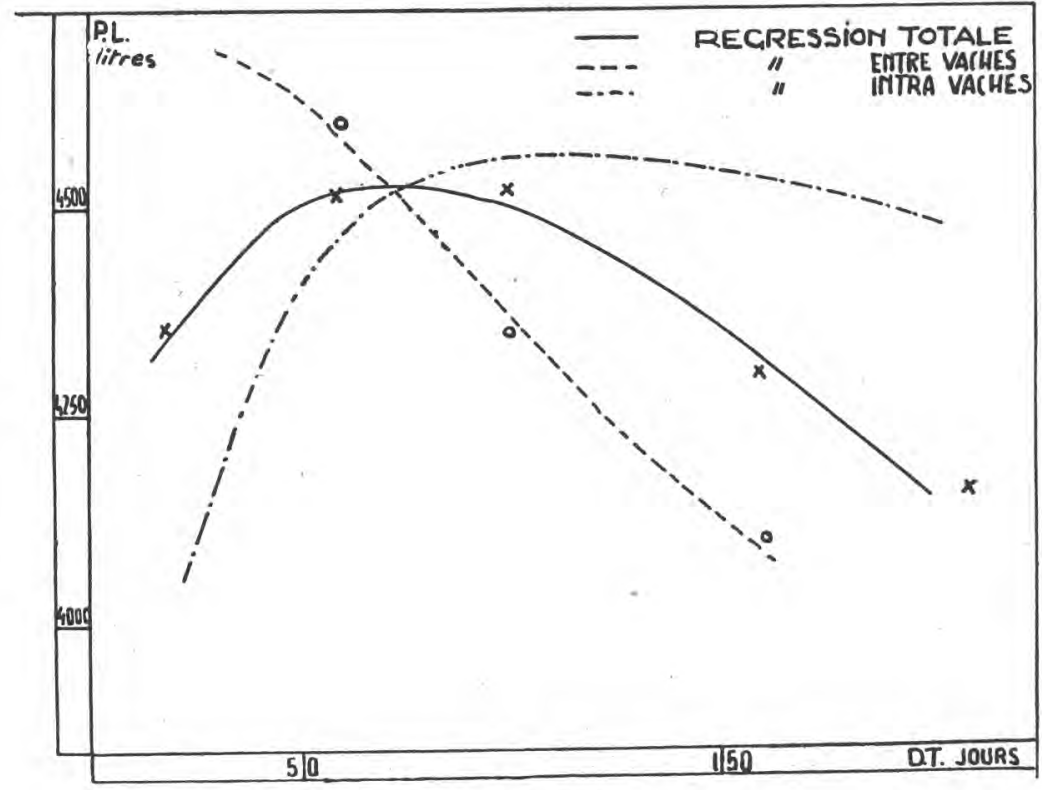

Fig. 5. - Nos courbes

une représentation hypothétique de la variation intra-vaches, qui traduit pour l'animal-type du troupeau les relations existant 
en moyenne, entre ses D. T. précédentes et ses P. L. correspondantes. Les coefficients de régression signalés précédemment confirment les différences d'allure des trois courbes :

- la courbe de variation totale s'apparente bien à celle qui a été signalée par les auteurs qui, comme LöRTSCHER, ont étudié cette relation sur un échantillon quelconque de données;

- la courbe de régression entre vaches traduit une influence génétique; les fortes productrices ont en moyenne une période sèche plus courte que les mauvaises productrices. Ce résultat est-il lié à la persistance ou au fait qu'en fin de lactation, les fortes productrices fournissent encore trop de lait ?

- la relation intra-vaches traduit l'incidence physiologique du repos mammaire sur la qualité de lait produite au cours de la lactation suivante, la courbe obtenue est sensiblement voisine de celles qui ont été rapportées par SANDERs d'une part, JoHANSSON et HaNsson d'autre part.

On peut dégager de cette constatation une règle d'exploitation moyenne pour une vache-type de la population que nous avons étudiée : il est inutile de prolonger les périodes sèches des laitières au-delà de quatre-vingts jours.

\section{IV. - Conclusion}

L'étude de l'influence de la D. T. sur la P. L. suivante, nous aura une fois de plus montré les difficultés rencontrées dans de telles recherches. Une analyse statistique pour être particulièrement concluante devrait toujours porter sur un matériel animal parfaitement connu et homogène en ce qui concerne les facteurs de variabilité autres que les facteurs étudiés.

Il existe, en fait, des interactions nombreuses entre tous les paramètres, tant du cycle de production (durée de tarissement, durée de lactation), que du cycle de reproduction (intervalle vêlage-fécondation ou intervalle entre vêlages), qui influencent la production laitière et de matière grasse de nos animaux. Ces dernières sont elles-mêmes fortement contingentes de facteurs purement extrinsèques, tels que le mois de vêlage par exemple.

Des recherches complémentaires sur les variations de l'influence de la durée de tarissement avec le niveau de production des femelles s'avéreraient nécessaires; il serait bon d'étudier également les répercussions de la durée de tarissement sur le détail des performances suivantes : production maximum, date à laquelle ce maximum est atteint, variabilité du taux butyreux au cours des trois premiers mois de la lactation; on sait en effet qu'elle est l'importance d'un "steaming" bien conduit avant vêlage. Les femelles en fin de gestation sont généralement aptes à constituer des réserves. 
En relation avec ce dernier point, des données plus précises sur l'alimentation des vaches, et notamment sur leur alimentation minérale pendant la période sèche, seraient intéressantes à recueillir systématiquement. L'exploration phosphocalcique du poil [1] à la fin du tarissement, pourrait peut être se révéler un test biologique satisfaisant des conditions de nutrition minérale pendant cette période.

Entre vaches, se dégage nettement la tendance à ce que les sujets les plus forts producteurs aient en même temps la durée de tarissement la plus courte.

Par contre, pour une vache moyenne de la population, on peut dire qu'au delà de quatre-vingts jours, une augmentation de la durée de sa période sèche, n'est pour elle d'aucune utilité zooteehnique pour sa lactation suivante. SANDERS et HAMmoND [3], concluaient d'une manière analogue.

(Travail en collaboration: Laboratoire de Zootechnie de l'Institut National agronomique, Professeur A. M. Leroy et Centre de Biologie du Conservatoire National des Arts et Métiers, Professeur H. Heim de Balsac.)

\section{RÉFÉRENCES BIBLIOGRAPHIQUES}

[1] M. Brochart. L'exploration de la nutrition phospho-calcique des vaches laitières au moyen du dosage de Ca et de $\mathrm{P}$ dans les poils. C. R. Acad. Agr., 1956, no 13, 663-675.

[2] E. B. Forbes et F. M. BeEgre. The mineral metabolism of the dairy cow. Ohio Agr. Exp. St. Bull., 1916, 295.

[3] J. HAMmond et H. G. SANDers. Some factors affecting milk yield. The J. of Agr. Sc., 1923, 13, 100.

[4] I. Johansson et A. Hansson. Causes of variation in milk and butterfat yield of dairy cows. Kungl. Lantbruksakad. Tid., 1940, no 6, 40-52.

[5] A. M. Leroy. Le producteur de lait. Encyclopédie des connaissances agricoles. Hachette, 200-202.

[6] H. Lörtscher. Variations statistisehe Untersuchungen an Ieistungserhebungen in einer British Friesian Herde. Zeits. f. Tierzüch. und Züchtungsbiol. 1937, 39, $\mathrm{n}^{\circ} 3,285-286$.

[7] J. Pouy et Coll. Evolution de la production laitière, du taux butyreux et de la production de matière grasse des vaches avec le numéro d'ordre de leur lactation. (Travaux non publiés.)

[8] J. Poly et Coll. Influence du mois de vêlage sur la production des vaches laitières en Seine-et-Marne. (Travaux non publiés.)

[9] H. G. SANDERs. The variations in milk yields, caused by season of the year, service, age and dry period, and their élimination. Part IV : Dry Period and Standardisation of yields. The J. of Agr. Sc., 1928, 18, 216-234.

[10] E. W. Swanson. The effect of fattening dairy heifers-upon their growth and lactation. The J. of Dairy Sc., 1957, 40, n ${ }^{\circ} 6,611$. 